LA W RENCE LIVERMORE N A TIO NAL LABORATORY

\title{
First Physics from HotQCD Collaboration
}

Ron Soltz, Rajan Gupta, Jeff Grandy

November 6, 2006 
This document was prepared as an account of work sponsored by an agency of the United States Government. Neither the United States Government nor the University of California nor any of their employees, makes any warranty, express or implied, or assumes any legal liability or responsibility for the accuracy, completeness, or usefulness of any information, apparatus, product, or process disclosed, or represents that its use would not infringe privately owned rights. Reference herein to any specific commercial product, process, or service by trade name, trademark, manufacturer, or otherwise, does not necessarily constitute or imply its endorsement, recommendation, or favoring by the United States Government or the University of California. The views and opinions of authors expressed herein do not necessarily state or reflect those of the United States Government or the University of California, and shall not be used for advertising or product endorsement purposes.

This work was performed under the auspices of the U.S. Department of Energy by University of California, Lawrence Livermore National Laboratory under Contract W-7405-Eng-48. 


\section{First physics from HotQCD collaboration}

The following pages show results from the first series of runs on BG/L using the unoptimized code from the MILC collaboration. The calculations were run with the AsqTad improved staggered fermion action and the RHMC algorithm on a $32^{3} \mathrm{x} 8$ lattice.

The jobs were run mostly during the October, 2006 on approximately $5 \%$ of the machine. The run consisted of approximately 1000 trajectories per beta value, spanning beta $=6.458$ to 6.85 , covering a temperature range of $140-210 \mathrm{MeV}$.

The analysis was performed using scripts provided by Carleton DeTar. Highlight marks on the scanned output show the values of the chiral condensate (green) and polyakov loop (pink). 
$\mathrm{Nt}=8 \quad\left(\mathrm{~m}_{-}\{u, d\}=0.1 \mathrm{~ms}\right)$ RHMC

beta mu,d ms $u \emptyset$ ns nt ReP dReP chi_L dchi_L cgiter dcgiter pbp_ud dpbp_ud pbp_s dpbp_s plaq dplaq rect drect pgm dpgm pb_dmdu_p_ud dpb_dmdu_p_ud pb_dmdu_p_s dpb_dmdu_p_s dS1 ddS1 dt accept N logfile $6.45800 .00500 \quad 0.0500 \quad 0.854932$

$\begin{array}{lllllllllllll}8 & 0.0024 & 0.0002 & 0.061 & 0.004 & 0.0 & 0.0 & 0.07995 & 0.00018 & 0.1940 & 0.0001 & 1.60249\end{array}$ $\begin{array}{lllllll}0.00008 & 0.84230 & 0.00011 & 0.86229 & 0.00013 & -4.89294 & 0.00025\end{array}$

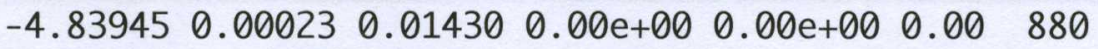

0328f21b6458m00820m0820r.00115_01100

$6.5000 \quad 0.00500 \quad 0.0500 \quad 0.856932$

$\begin{array}{lllllllllllll}8 & 0.0032 & 0.0003 & 0.063 & 0.003 & 0.0 & 0.0 & 0.06690 & 0.00021 & 0.1755 & 0.0002 & 1.61820\end{array}$ $\begin{array}{lllllll}0.00016 & 0.86239 & 0.00020 & 0.88358 & 0.00020 & -4.88984 & 0.00036\end{array}$

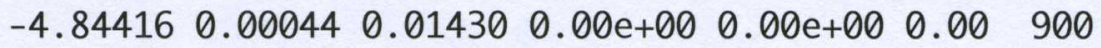

o328f21b650m00765m0765r.00125_01120

$6.5500 \quad 0.00500 \quad 0.0500 \quad 0.859432$

$8 \quad 0.0047 \quad 0.0005 \quad 0.074 \quad 0.006 \quad 0.0 \quad 0.0 \quad 0.05430 \quad 0.00023 \quad 0.1561 \quad 0.00021 .63616$ $\begin{array}{lllllll}0.00014 & 0.88538 & 0.00019 & 0.90795 & 0.00022 & -4.88030 & 0.00042\end{array}$

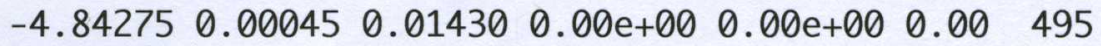

0328f21b655m00705m0705r.00160_00720

$6.6000 \quad 0.00500 \quad 0.0500 \quad 0.861632$

$\begin{array}{lllllllllllll}8 & 0.0074 & 0.0005 & 0.068 & 0.004 & 0.0 & 0.0 & 0.04182 & 0.00018 & 0.1377 & 0.0001 & 1.65320\end{array}$

$\begin{array}{lllllll}0.00007 & 0.90737 & 0.00010 & 0.93115 & 0.00011 & -4.87391 & 0.00018\end{array}$

$\begin{array}{llllll}-4.84310 & 0.00019 & 0.01430 & 0.00 \mathrm{e}+00 & 0.00 \mathrm{e}+00 & 0.00 \quad 925\end{array}$

o328f21b660m00650m0650r.00145_01145

$6.6500 \quad 0.00500 \quad 0.0500 \quad 0.863632$

$\begin{array}{lllllllllllll}8 & 0.0131 & 0.0008 & 0.075 & 0.007 & 0.0 & 0.0 & 0.03116 & 0.00052 & 0.1215 & 0.0003 & 1.66904\end{array}$

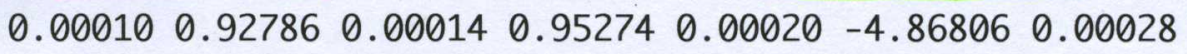

$\begin{array}{llllll}-4.84283 & 0.00040 & 0.01430 & 0.00 \mathrm{e}+00 & 0.00 \mathrm{e}+00 & 0.00 \quad 510\end{array}$

o328f21b665m00599m0599r.00115_00730

$6.7000 \quad 0.00500 \quad 0.0500 \quad 0.865732$

$\begin{array}{llllllllllll}8 & 0.0191 & 0.0007 & 0.073 & 0.004 & 0.0 & 0.0 & 0.02109 & 0.00066 & 0.1062 & 0.0003 & 1.68470\end{array}$

$\begin{array}{lllllll}0.00006 & 0.94827 & 0.00008 & 0.97417 & 0.00010 & -4.85880 & 0.00023\end{array}$

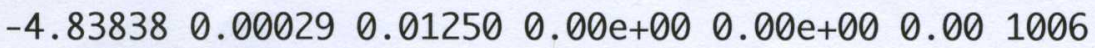

o328f21b670m00552m0552r.00269_01275

$6.7600 \quad 0.00500 \quad 0.0500 \quad 0.867832$

$\begin{array}{llllllllllll}8 & 0.0276 & 0.0003 & 0.077 & 0.005 & 0.0 & 0.0 & 0.01332 & 0.00018 & 0.0905 & 0.0001 & 1.70190\end{array}$

$\begin{array}{lllllll}0.00005 & 0.97067 & 0.00008 & 0.99759 & 0.00009 & -4.85147 & 0.00015\end{array}$

$\begin{array}{llllll}-4.83587 & 0.00017 & 0.01250 & 0.00 \mathrm{e}+00 & 0.00 \mathrm{e}+00 & 0.00 \quad 995\end{array}$

0328f21b676m005m05r.00217_01215

$6.8000 \quad 0.00500 \quad 0.0500 \quad 0.869232$

$\begin{array}{lllllllllllll}8 & 0.0333 & 0.0005 & 0.085 & 0.012 & 0.0 & 0.0 & 0.01051 & 0.00018 & 0.0820 & 0.0002 & 1.71298\end{array}$

$0.00007 \quad 0.985160 .000091 .012760 .00009-4.845320 .00015$

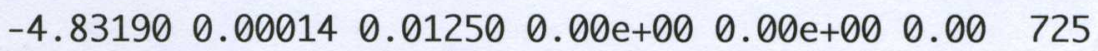

o328f21b680m00471m0471r.00205_00950

$6.8500 \quad 0.00500 \quad 0.0500 \quad 0.870932$ 
$\begin{array}{lllllllllllll}8 & 0.0396 & 0.0006 & 0.086 & 0.007 & 0.0 & 0.0 & 0.00842 & 0.00011 & 0.0727 & 0.0001 & 1.72655\end{array}$

$0.000061 .002950 .000071 .031230 .00008-4.83754 \quad 0.00014$

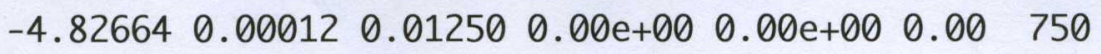

o328f21b685m00437m0437r.00170_00970 